\section{NKG2D-based chimeric antigen receptor therapy induced remission in a relapsed/refractory acute myeloid leukemia patient}

Acute Myeloid Leukemia (AML) is the most common acute leukemia affecting adults characterized by the accumulation of immature myeloblasts in the marrow or peripheral blood. Natural Killer Group 2D (NKG2D) is an activating receptor expressed on Natural Killer (NK) cells and activated $\mathrm{CD} 8^{+} \mathrm{T}$ cells which triggers cytotoxicity following recognition of its ligands (namely MHC class I polypeptide-related sequence $A$ and $B$ (MICA/B) and unique long protein 16 (UL-16) binding protein 1 to 6 (ULBP1 to 6)) at the surface of stressed, infected and most notably transformed cells. ${ }^{1}$ Consequently, a broad range of primary tumors express NKG2D ligands (NKG2DL) and can be targeted by NKG2D-based immunotherapies. ${ }^{2,3}$ NKG2DL expression was reported in AML, with data reporting $67 \%$ to $100 \%$ expression in MICA/B and/or ULBP2-3 expression in blasts ${ }^{4,5}$ with all blasts expressing at least one ligand [Dulphy $N$, Toubert A et al., unpublished data].

CYAD-01 are autologous $T$ cells genetically modified to express a chimeric antigen receptor (CAR) comprising a fusion of the human full-length NKG2D receptor with the CD3 3 signaling domain. ${ }^{6}$ Although the co-stimulato- ry molecule DNAX-activating protein 10 (DAP10) is not part of the transgene, NKG2D associates with this molecule for membrane stabilization to provide the secondary activation signal. ${ }^{1}$ The NKG2D-CAR construct binds 8 different ligands in an MHC-independent fashion expressed by cancer cells of diverse origins. ${ }^{3,7,8}$ Because the ligands for the NKG2D receptor are absent or expressed at very low levels in normal tissues, ${ }^{1,9}$ specificity for tumors is considered to be high. In agreement, we previously demonstrated no recognition of normal peripheral blood mononuclear cells nor healthy bone marrow from healthy patients by CYAD-01 in vitro. ${ }^{10}$ Preclinical studies have shown that CYAD-01 mediates potent anti-tumor activity against both hematological and solid tumors without the requirement of prior lymphodepleting chemotherapy., ${ }^{3,6}$ Single intravenous administration of low dose autologous CYAD-01 (maximum $3 \times 10^{7}$ flat dose), without prior chemotherapy, has been tested in a completed Phase I study (clinicaltrials.gov identifier 02203825) which evaluated 12 patients with relapsed/refractory AML (r/r AML), myelodysplastic syndrome (MDS), or multiple myeloma (MM). ${ }^{11}$ The preliminary data obtained suggested encouraging signs of activity with one AML patient treated at the $3 \times 10^{7}$ CYAD-01 dose-level having hematologic improvement for three months post-CYAD-01 treatment. ${ }^{11}$ Building on these initial results and preclinical data supporting multiple
A

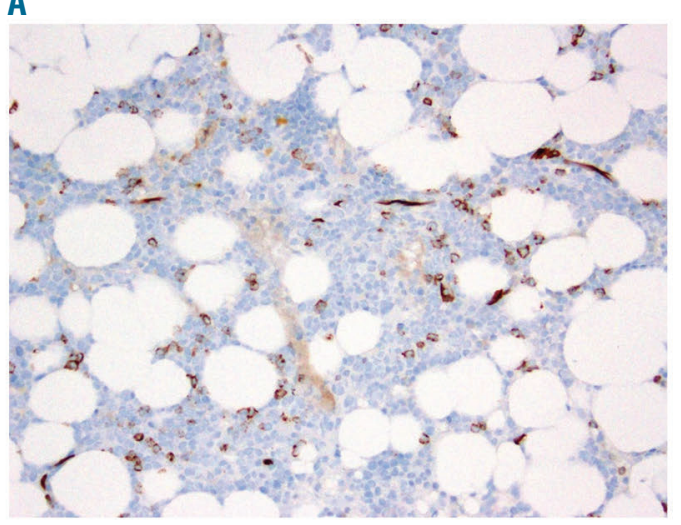

C

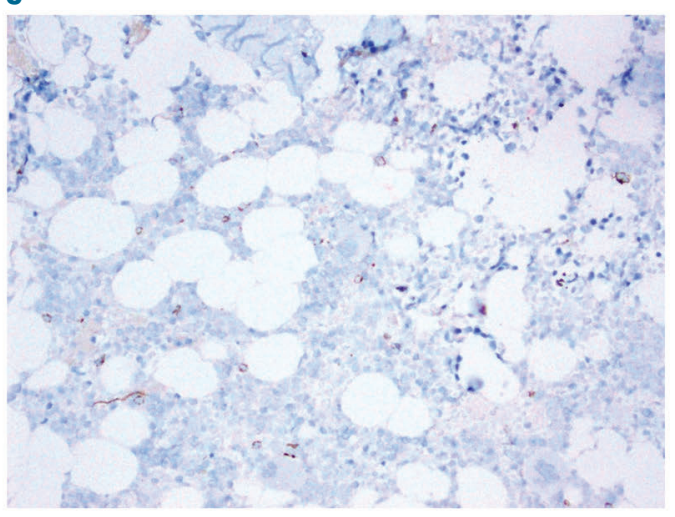

B
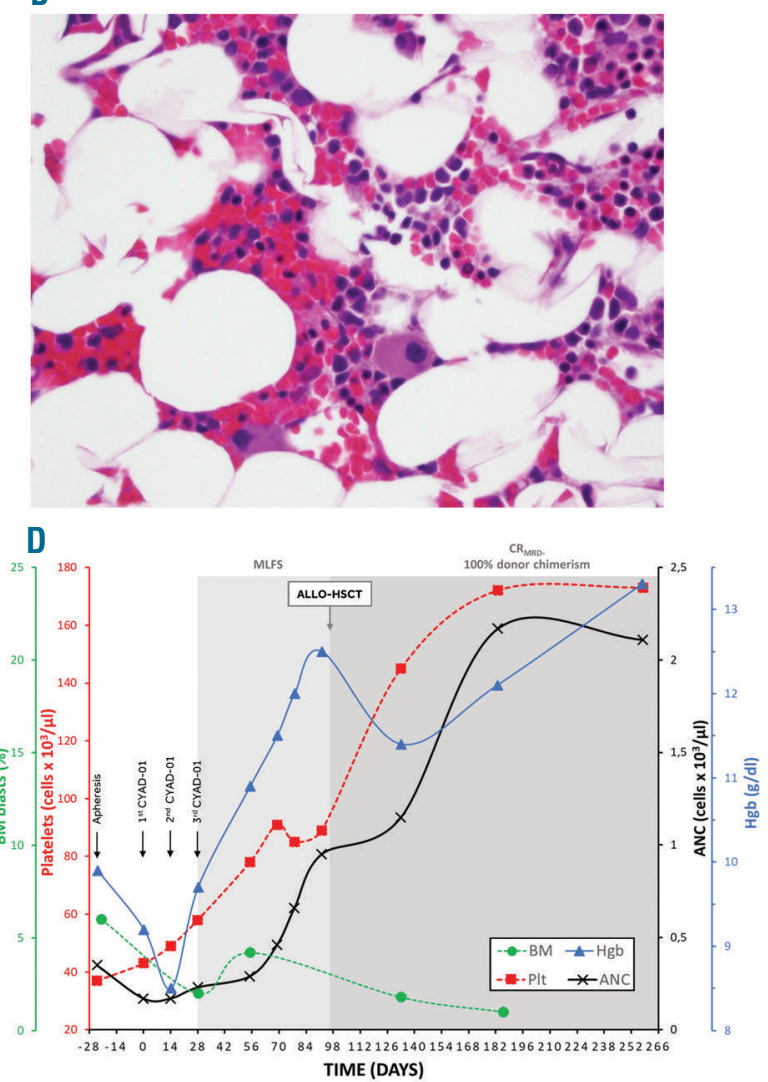

Figure 1. Relapsed AML patient treated with CYAD-01 CAR-T therapy. CD34 IHC of the core biopsy shows clusters of blasts ( 7\%) consistent with relapsed disease (Panel A). Prominent dysmegakaryopoiesis was also noted (Panel B). BM biopsy at day +56 following 3 infusions of CYAD-01 showed normal trilineage hematopoiesis with CD34 IHC showing no increase in blasts (Panel C). Patient also with significant improvement of hematopoiesis as transfusion independent with marked increase of hemoglobin and platelet count (Panel D). 
CYAD-01 infusions are necessary for disease eradication; we initiated the THINK study (clinicaltrials.gov identifier 03018405) evaluating the safety and clinical activity of multiple treatment administrations of CYAD-01 in different solid and hematological indications. ${ }^{6}$

We report here the case of a 52-year-old male with $\mathrm{r} / \mathrm{r}$ AML. The patient had +8/del(7)(q22q36), FLT3/NPM1 wild-type AML that was primary refractory to induction with $7+3$ with daunorubicin and cytarabine. The patient subsequently received salvage chemotherapy with cladribine, cytarabine, G-CSF and mitoxantrone (CLAG$\mathrm{M})$, and achieved CR1 followed by two cycles of CLA consolidation. Following a 7-month remission where allogeneic hematopoietic stem cell transplantation (alloHSCT) was delayed to allow for pulmonary function test recovery, the patient had recurrent cytopenias and bone marrow (BM) biopsy confirmed relapsed disease with hypocellularity, $7 \%$ blasts in numerous clusters and dysmegakaryopoiesis (Figure 1A and $1 \mathrm{~B}$ ). The patient developed severe constitutional symptoms with fatigue, anorexia, body pain and drenching night sweats. Given no standard treatment options, the patient enrolled in the THINK trial and underwent apheresis followed by CYAD-01 infusions at the initial dose level of $3 \times 10^{8}$ cells (flat dose)/injection every 2 weeks for 3 administrations without any lymphodepleting chemotherapy. ${ }^{6} \mathrm{CYAD}-01$ was well tolerated with non-related grade 1 adverse events without cytokine release syndrome (CRS) or neurotoxic effects. BM evaluation at day +28 following 2 CYAD-01 infusions showed morphologic leukemia-free state (MLFS, $2 \%$ blasts) with normocellularity and trilineage hematopoiesis, which was confirmed at day +56 $\mathrm{BM}$ with $\operatorname{del}(7 \mathrm{q})$ by FISH at $7 \%$ (Figure $1 \mathrm{C}$ ). Serial next- generation sequencing (NGS) of BM-MNCs showed persistent DNMT3A R882H mutation, but also identified a new IDH2 R172K mutation. The patient had resolution of symptoms with improved hematopoiesis, which continued to improve until the patient was treated with an allo-HSCT on day +97 post CYAD-01 (Figure 1D). At disease evaluation 100 days post allo-HSCT (day +197 post CYAD-01), the patient achieved a complete molecular remission by serial NGS with $100 \%$ donor chimerism (Figure 1D). To date, the patient has maintained a CR 6 months from allo-HSCT and 9 months following initial CYAD-01 infusion. Figure 2A shows timeline of the patient's history of treatments and responses.

Molecular analysis indicated a low level of CYAD-01 cells in the periphery until day +4 post injection. The patient CYAD-01 product primarily consisted of effectormemory phenotype $\mathrm{CD}^{+} \mathrm{T}$ cells with robust target celldirected cytotoxicity and interferon (IFN)- $\gamma$ secretion. (Online Supplementary Figure $S 1 A$ and $S 1 B$ ). Immunohistochemistry (IHC) analysis on the baseline BM taken showed positive staining of foci of neoplastic cells for all NKG2DL assessed (Online Supplementary Figure S2), with the strongest expression for MICA/MICB (Figure 2B). Of note, there was no NKG2DL expression on the non-neoplastic BM cells. Finally, it is important to mention no detectable level of the transgene was evidenced at day $+1,+4$ and +32 post-injection, using a Vector copy number (VCN) qPCR-based assay, suggesting poor in vivo expansion and persistence of the injected cell product.

CAR therapy has brought forth a paradigm change in the management of refractory chemo-resistant B-cell leukemia. ${ }^{12}$ However, the choice of target in AML has

A

\begin{tabular}{|c|c|c|c|c|c|c|c|c|c|c|c|c|c|c|c|c|c|c|c|c|}
\hline \multicolumn{6}{|c|}{2016} & \multicolumn{12}{|c|}{2017} & \multicolumn{3}{|c|}{2018} \\
\hline Jul & Aug & Sep & Oct & Nov & Dec & Jan & Feb & Mar & Apr & May & Jun & Jul & Aug & Sep & Oct & Nov & Dec & Jan & Feb & Mar \\
\hline \multicolumn{6}{|c|}{ AML diagnosed CR1 } & & & & \multicolumn{3}{|c|}{ Relapse } & \multicolumn{3}{|c|}{ MLFS } & \multicolumn{3}{|c|}{ CR MRD- } & & & \\
\hline & $7+3 \mathrm{CL}$ & G-M C & $\mathrm{LA}$ & & & & & & & & & $\overrightarrow{C Y A D}$ & & & ${ }_{\mathrm{O}-\mathrm{HSCT}}$ & & & & & \\
\hline
\end{tabular}

B

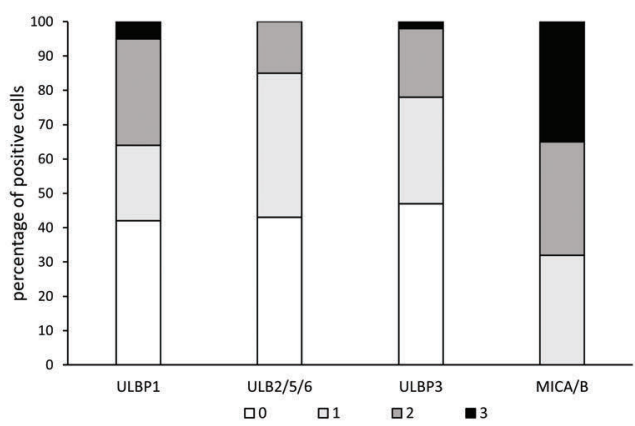

C

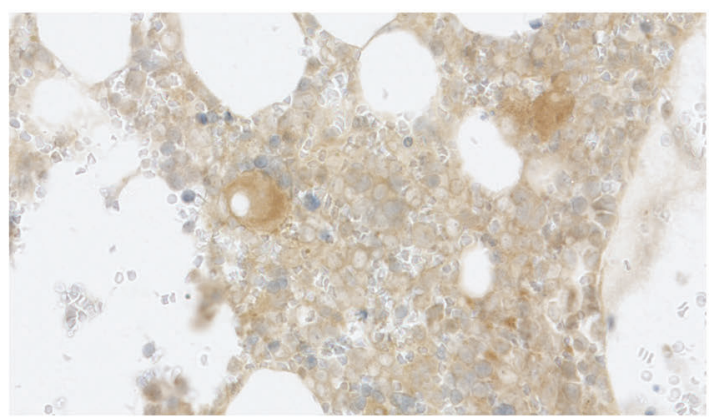

Figure 2. Treatment Course and immunohistochemistry analysis of NKG2D ligand expression in bone marrow sample taken before first CYAD-01 injection. Timeline of history of treatments and responses is shown (A). BM biopsy of patient prior to CYAD-01 treatment was subject to a range of IHC staining protocols specific for the following NKG2DL: MICA/MICB, ULBP1, ULBP2/5/6, ULBP3. In addition, a sample of each was prepared applying standard hematoxylin and eosin (H\&E) staining (data not shown). All samples were examined and graded by light microscopy. 100 presumptive neoplastic cells were counted and analysed, in terms of staining characteristics, using a "H-score" approach. The intensity of membranous staining was graded for each cell, as follows: 0 - no staining; 1 - minimal staining (each positive cell scored “1"); 2 - mild staining (each positive cell scored "2"); 3 - moderate/marked staining (each positive cell scored "3"). The percentage of positive cells for each intensity staining and each ligand is represented in (B). Representative staining of MICA/B is shown in (C). 
been challenging due to their normal expression on hematopoietic stem cells leading to potential irreversible hematopoietic toxicity. ${ }^{13}$ Thus, current CAR-T strategies in AML either require a backup allo-HSCT or unproven shut-off mechanisms. ${ }^{14}$ In contrast, NKG2DL are upregulated in transformed cells with minimal expression in normal tissues. ${ }^{1}$ This was confirmed by the absence of significant adverse events of CYAD-01 in this AML patient and no Grade 3 or above adverse events related to treatment in any of the AML patients enrolled in the THINK trial up to now. This is of critical clinical importance given CD19 specific CAR T-cell therapies with grade 5 toxicities including CRS, neurotoxicity and ontarget off-tumor toxicity against normal cells. ${ }^{15}$ The low toxicity, with clinical activity, observed in this patient is most probably related to multiple factors: (i) CYAD-01 cells demonstrate only short-term persistence and low proliferation post-infusion, (ii) the chimeric CYAD-01 construct consists entirely of human sequences, with an entirely native extracellular domain not expected to induce the anaphylaxis reactions observed with other CAR-Ts using scFv from murine origin, ${ }^{16}$ and (iii) the absence of previous lymphodepleting chemotherapy.

While reduction of blasts occurred in AML patients treated with LeY-specific CAR-Ts was also previously reported, the clinical response could not be entirely attributed to the activity of the CAR-T since these patients were in morphological CR after reinduction with fludarabine prior to CAR-T administration. ${ }^{17}$ In the only published $\mathrm{r} / \mathrm{r}$ AML case report evaluating the CD33-specific CAR-Ts without conditioning therapy, a 2-week transient decrease in blast count was reported in the context of grade 4 chills, fevers and transient hyperbilirubinemia, but with a rapid disease progression, suggesting tumor escape mechanism. ${ }^{18}$ Of clinical interest in the setting of MLFS and blood counts approaching complete remission at the time of allo-HSCT, the patient had identification of a IDH2 mutation that was not present prior to treatment. This suggests that there can be heterogeneity of NKG2D ligand expression that is dependent on the underlying molecular architecture. This is supported by a recent study in $I D H$ mutant gliomas where NKG2D ligands were down-regulated. ${ }^{19}$ Future study is required to further investigate molecular drivers of NKG2D ligand expression in AML in order to further augment efficacy of CYAD-01. In summary, we report the first objective response to CAR-T in $\mathrm{r} / \mathrm{r}$ AML using CYAD-01 without preconditioning chemotherapy and with no significant toxicities, highlighting the potential of targeting NKG2DL in AML.

David A. Sallman, Jason Brayer, ${ }^{1}$ Elizabeth M. Sagatys, Caroline Lonez, Eytan Breman, Sophie Agaugué, ${ }^{3}$

Bikash Verma, ${ }^{4}$ David E. Gilham, ${ }^{3}$ Frédéric F. Lehmann ${ }^{3}$ and Marco L Davila

${ }^{1}$ Malignant Hematology and 'Hematopathology and Laboratory Medicine, H. Lee Moffitt Cancer Center and Research Institute, Tampa, FL, USA; ${ }^{3}$ Celyad, SA, Mont-Saint-Guibert, Belgium; ${ }^{4}$ Celyad, SA, New York, NY, USA and 'Blood \& Marrow Transplantation and Cellular Immunotherapy, H. Lee Moffitt Cancer
Center and Research Institute, Tampa, FL, USA

$D A S$ and $J B$ contributed equally to this work.

Correspondence:David.Sallman@moffitt.org/

Marco.Davila@moffitt.org

doi:10.3324/haematol.2017.186742

Information on authorship, contributions, and financial \& other disclosures was provided by the authors and is available with the online version of this article at www. haematologica.org.

\section{References}

1. Raulet DH, Gasser S, Gowen BG, Deng W, Jung H. Regulation of ligands for the NKG2D activating receptor. Annu Rev Immunol. 2013; 31:413-441.

2. Spear P, Wu M-R, Sentman M-L, Sentman CL. NKG2D ligands as therapeutic targets. Cancer Immun. 2013;13:8.

3. Demoulin B, Cook WJ, Murad J, et al. Exploiting natural killer group 2D receptors for CAR T-cell therapy. Future Oncol. 2017; 13(18):1593-1605

4. Hilpert J, Grosse-Hovest L, Grunebach F, et al. Comprehensive analysis of NKG2D ligand expression and release in leukemia: implications for NKG2D-Mediated NK cell responses. J Immunol. 2012; 189(3):1360-1371.

5. Nowbakht P, Ionescu M-CS, Rohner A, et al. Ligands for natural killer cell-activating receptors are expressed upon the maturation of normal myelomonocytic cells but at low levels in acute myeloid leukemias. Blood. 2005;105(9):3615-3622.

6. Lonez C, Verma B, Hendlisz A, et al. Study protocol for THINK: a multinational open-label phase I study to assess the safety and clinical activity of multiple administrations of NKR-2 in patients with different metastatic tumour types. BMJ Open. 2017;7(11):e017075.

7. Sentman CL, Meehan KR. NKG2D CARs as cell therapy for cancer. Cancer J. 2014;20(2):156-159.

8. Le Bert N, Gasser S. Advances in NKG2D ligand recognition and responses by NK cells. Immunol Cell Biol. 2014;92(3):230-236.

9. Raulet DH. Roles of the NKG2D immunoreceptor and its ligands. Nat Rev Immunol. 2003;3(10):781-790.

10. Barber A, Zhang T, Megli CJ, Wu J, Meehan KR, Sentman CL. Chimeric NKG2D receptor-expressing $T$ cells as an immunotherapy for multiple myeloma. Exp Hematol. 2008;36(10):1318-1328.

11. Nikiforow S, Murad J, Daley H, et al. A first-in-human phase I trial of NKG2D chimeric antigen receptor-T cells in AML/MDS and multiple myeloma. J Clin Oncol. 2016;34(15 Suppl):TPS3102.

12. Maude SL, Frey N, Shaw PA, et al. Chimeric antigen receptor T cells for sustained remissions in leukemia. $N$ Engl J Med. 2014;371(16):1507-1517.

13. Mardiros A, Forman SJ, Budde LE. T cells expressing CD123 chimeric antigen receptors for treatment of acute myeloid leukemia. Curr Opin Hematol. 2015;22(6):484-488.

14. Fan M, Li M, Gao L, et al. Chimeric antigen receptors for adoptive $\mathrm{T}$ cell therapy in acute myeloid leukemia. J Hematol Oncol. 2017;10(1):151.

15. Brudno JN, Kochenderfer JN. Toxicities of chimeric antigen receptor T cells: recognition and management. Blood. 2016;127(26):33213330.

16. Lamers $\mathrm{CH}$, Willemsen $\mathrm{R}$, van Elzakker $\mathrm{P}$, et al. Immune responses to transgene and retroviral vector in patients treated with ex vivo-engineered T cells. Blood. 2011;117(1):72-82.

17. Ritchie DS, Neeson PJ, Khot A, et al. Persistence and efficacy of second generation CAR T cell against the LeY antigen in acute myeloid leukemia. Mol Ther. 2013;21(11):2122-2129.

18. Wang OS, Wang Y, Lv HY, et al. Treatment of CD33-directed chimeric antigen receptor-modified $T$ cells in one patient with relapsed and refractory acute myeloid leukemia. Mol Ther. 2015;23(1):184-191.

19. Zhang X, Rao A, Sette P, et al. IDH mutant gliomas escape natural killer cell immune surveillance by downregulation of NKG2D ligand expression. Neuro Oncol. 2016;18(10):1402-1412. 